\title{
Assessment of Environmental Literacy
}

\author{
Alexandre Dias ${ }^{1}$, Margarida Figueiredo ${ }^{1,2}$, Humberto Chaves $^{3}$, José Neves ${ }^{4,5}$, Henrique \\ Vicente $^{1,4,6}$
}

${ }^{1}$ Departamento de Química, Universidade de Évora, Portugal, ${ }^{2}$ CIEP, Universidade de Évora, Portugal, ${ }^{3}$ Escola Superior Agrária de Beja, Instituto Politécnico de Beja, Portugal ${ }^{4}$ Centro Algoritmi, Universidade do Minho, Portugal, ${ }^{5}$ Instituto Politécnico de Saúde do Norte, CESPU, Portugal, ${ }^{6}$ REQUIMTE/LAQV, Universidade de Évora, Portugal.

\begin{abstract}
Nowadays, the issues related with environment preservation assume an increasing importance. Progressively, more sustainable solutions/techniques are being developed to combat environmental destruction. The decision to include themes related to the environment in the curriculum of technological courses in higher education aims to promote more sustainable behaviors and in an indirect way, increase the environmental literacy of the population. Thus, this study aims to evaluate the environmental literacy focusing on four topics, i.e., air pollution, water pollution, global warming, and energy resources. For this purpose, a questionnaire was developed and applied to a convenience sample, formed by individuals of both genders, aged between 20 and 81 years old. The questionnaire intended to collect data to characterize the sample and assess the literacy regarding environmental issues. In order to carry out the environmental literacy assessment, the respondents were asked to express their degree of agreement with some statements related with the environmental themes mentioned above. The data collected was analyzed using data mining tools. The results suggest that the population's literacy is satisfactory in relation to some issues, but insufficient in relation to others, equally important, but less disseminated.
\end{abstract}

Keywords: Environmental literacy; sustainability; biotechnology; artificial neural networks. 


\section{Introduction}

Since the mid-eighties of the 20th century, mankind has repeatedly failed to achieve the goal of sustainability. Indeed, in the past few decades, highly developed human societies have consumed more resources than those the planet can produce, creating amounts of pollution far beyond the Earth's ability to absorb and purify (Wackernagel \& Rees, 1996). Despite the efforts of governments and various non-governmental organizations, the evidence shows that human efforts are not enough to prevent serious climate change or global warming. The efforts made are important, but they will not be enough if the population is not aware of the importance of being greener and kinder. This awareness requires an appropriate environmental education policy that mobilizes the population for a common goal - the sustainability of the Planet.

About 30 years ago, Dising \& Roth (1992) suggested that environmental literacy is the ability to understand and to interpret the relative balance of environmental systems and to take appropriate actions to maintain, restore or improve the health of those systems. Currently, the term environmental literacy comprises the knowledge and understanding of environmental concepts, problems and issues, affective and cognitive dispositions, as well as a set of competencies and skills, together with appropriate behavioral strategies to adopt relevant decisions in a wide range of environmental contexts (Hollweg et al., 2011). In simple terms, environmental literacy can be understood as the domain of four interrelated components: knowledge, attitudes, skills, and environmentally friendly behaviors (Hungerford \& Volk, 1990). Given that biotechnology is an emerging scientific field with extraordinary potential for creating innovative and environmentally friendly products and solutions that can make a vital contribution to global sustainability, it is particularly important to examine the level of population literacy on these issues.

Indeed, to ensure the success of any environmental policy, the population must adhere to it. For this it is necessary that the population knows and understands the underlying concepts and practices. Thus, this work intends to evaluate the environmental literacy in a small sample of the Portuguese population regarding to four main themes - air pollution, water pollution, global warming, and energy resources.

This paper comprises four sections. Following the introduction to the theme, the methodology for problem solving is presented (section 2), whereas the results are presented and discussed in section 3. Finally, in section 4, conclusions are drawn, and future work is outlined.

\section{Methods}

The data collection was carried out using the questionnaire survey technique. The questionnaire was evaluated by a set of specialists. After specialist analysis, the questionnaire 
was modified and applied to a restrict group of participants, not included in the sample. Due to the pandemic of the new coronavirus (COVID-19) and all associated changes in society's daily life, the questionnaire was distributed randomly in digital form.

The questionnaire consists of two parts, the first referring to the collection of sociodemographic data (e.g., age, gender, educational qualifications, and place of residence) and the second containing a set of statements on which respondents were asked to give their opinion on the four topics covered (i.e., air pollution, water pollution, global warming, and energy resources). In the first section, the answers are descriptive while the second used a Likert scale with five levels (i.e., strongly agree, agree, disagree, strongly disagree and I don't know).

The former topic comprises the statements, viz.

S1 Reducing the use of combustion engines helps to reduce air pollution;

S2 The use of activated carbon contributes to the retention of polluting gases; and

S3 The use of pesticides/fertilizers contributes to the air contamination.

The second topic encompasses the statements, viz.

S4 In oil spills in the oceans, the use of microorganisms accelerates the water purification;

S5 The use of specific plants along the watercourses helps the decontamination process; and

S6 The direct discharge of industrial waste into water courses causes the death of living organisms.

The topic regarding global warming includes the statements, viz.

S7 The use of fossil fuels as energy source is the main cause of global warming;

S8 The rapid glaciers melt is caused by the planet warming; and

S9 The use of large amounts of CFCs causes an increase in the ozone hole.

Finally, the topic concerning energy resources contains the statements, viz.

S10 The use of renewable energies contributes to the environment protection;

S11 Biogas is increasingly used as an energy source; and

S12 Renewable energies are inexhaustible.

With the aim of converting the qualitative data (collected via the questionnaire) into quantitative one, the method proposed by Fernandes et al. (2016) was applied. Thus, the set of $n$ statements relating to a particular topic is divided into a circle with an area of 1 (one) which is divided into $n$ slots, with the marks in the axis relating to each of the possible scale's options. The quantitative value corresponds to the total area according to Section 3.3. 
The Waikato Environment for Knowledge Analysis (WEKA) was used to set Artificial Neural Networks (ANNs) (Haykin, 2009) while maintaining the standard software parameters (Frank et al., 2016). In each simulation, the database was randomly divided into two mutually exclusive partitions, leading to the training test sets.

\section{Results and Discussion}

\subsection{Sample Characterization}

This study was carried out using a convenience sample that included 147 participants between 20 and 81 years of age, with an average of 35 . The gender distribution was $40.2 \%$ and $59.8 \%$ for male and female, respectively. In terms of academic qualifications, $14.3 \%$ of the cohort said they had a basic education, 51.0\% said they had completed secondary education, and $34.7 \%$ said they had a degree or postgraduate education. In terms of place of residence, $19.7 \%, 30.6 \%$ and $49.7 \%$ are from the northern, central, and southern regions of Portugal, correspondingly.

\subsection{Answer Frequency Analysis}

Figure 1 shows the frequency of answer to the second part of the questionnaire, where participants chosen the alternative that best express their opinion regarding each statement.

The statements $\mathrm{S} 1$ to $\mathrm{S} 3$ refer to the air pollution, $\mathrm{S} 4$ to $\mathrm{S} 6$ are related to water pollution, $\mathrm{S} 7$ to $\mathrm{S} 9$ are about global warming, and S10 to S12 are relative to energy resources. Regarding the statements included in the topic air pollution, $36.7 \%$ and $67.3 \%$ of respondents ticked the option agree, in statements 2 (related with the use of activated carbon) and 3 (concerning contamination by pesticides/fertilizers), respectively.

With regard to statement 1 (about the use of combustion engines), the majority of participants ticked the options strongly agree (52.3\%) and agree (44.9\%). The option disagree was chosen by $12.9 \%, 10.9 \%$ and $1.4 \%$ of participants in statements 2,3 and 1 , respectively. Regarding the option strongly disagree, it was not selected by the participants.

With regard to the topic water pollution all the participants chosen the options strongly agree $(67.3 \%)$ and agree $(32.7 \%)$ in statement 6 (related with direct discharge of industrial waste). Concerning statements 4 (related to the use of microorganisms for decontamination) and 5 (regarding the use of plants for decontamination) the option agree was the most marked (43.6\% and 51.0\% respectively) followed by the option strongly agree (18.4\% and $19.7 \%$ respectively). The option disagree was chosen by $10.9 \%$ and $1.4 \%$ of participants in statements 5 and 4, respectively. As before, the option strongly disagree was not selected.

Concerning the statements comprised in the topic global warming (i.e., statements S7 to S9), the option agree was the most chosen $(63.3 \%, 59.9 \%$ and $40.1 \%$ respectively) followed by 
the option strongly agree $(23.8 \%, 37.3 \%$ and $29.3 \%$ respectively). The option disagree was ticked only in statement 8 (related with glaciers melt), by $1.4 \%$ of participants. The option strongly disagree was not marked. Finally, with respect to the topic energy resources, $54.4 \%$ of participants ticked the option agree to statement 10 (related with the use of renewable energies), whereas $44.2 \%$ marked the option strongly agree.

Regarding statement 11 (about the use of biogas), $47.6 \%$ of participants chose the option agree, whereas only 5.5\% marked the option strongly agree. In statement 12 (on the inexhaustibility of renewable energies), $47.6 \%$ of participants chose the option agree, $15.0 \%$ ticked the option disagree, whereas the option strongly agree was marked by $12.9 \%$. It should be noted that $4.1 \%$ of respondents ticked the option strongly disagree.
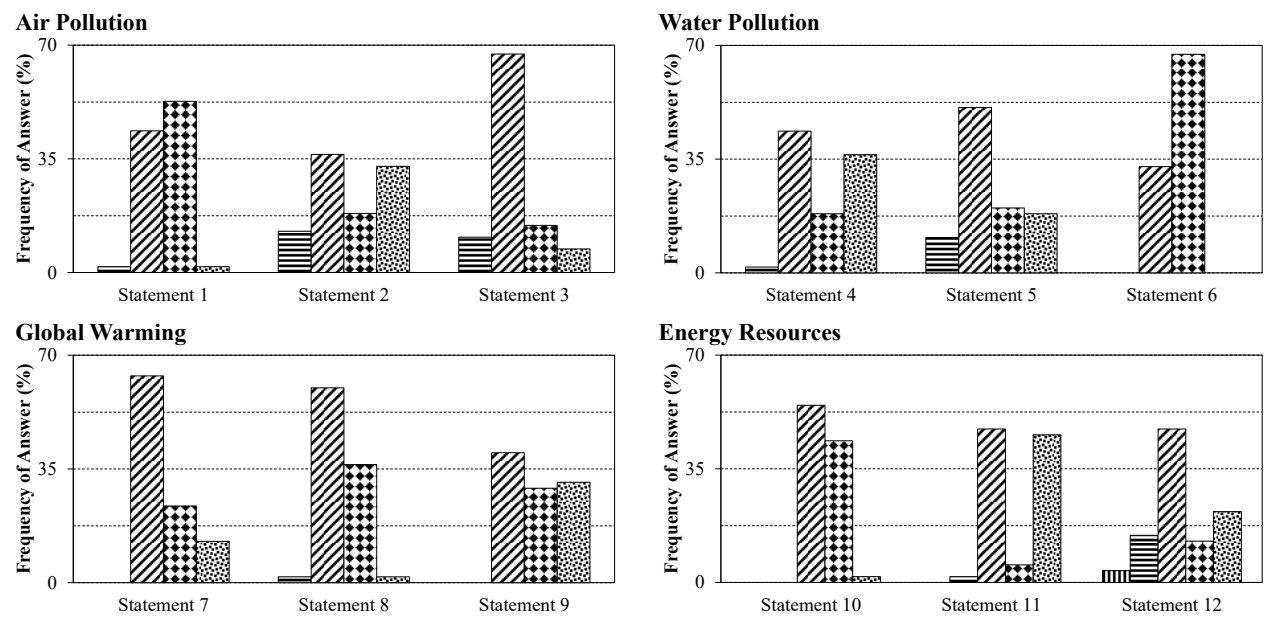

|fl||l| Strongly Disagree

Figure 1. Frequency of answer to the statements included in the second part of the questionnaire.

Regarding the option I don't know, the analysis of Figure 1 allows to identify 3 different situations, corresponding to frequencies of answer lesser than $2 \%$, ranging between $7 \%$ and $22 \%$, and higher than $30 \%$. The former group includes the statements 1 (about the use of combustion engines), 6 (related with direct discharge of industrial waste), 8 (related with glaciers melt) and 10 (related with the use of renewable energies).

The issues related with activated carbon (S2), microorganisms for decontamination (S4), CFCs (S9), and biogas (S11) integrate the third group, exhibiting frequencies of the answer I don't know higher than 30\%. This evidence can be justified considering that these issues are less disseminated through the media, with the population less informed about their importance. 


\subsection{Environmental Literacy Assessment}

To develop a decision support system for assessing the environmental literacy, ANNs were trained and tested on the basis of the results obtained in the second section of the questionnaire. Since the data collected is qualitative, it had to be quantified, being the method suggested by Fernandes et al. (2016) the one that was adopted. To illustrate the process, Table 1 presents the answers of participant 1 .

Table 1. The answers of participant 1 to the statements included in questionnaire ( $2^{\text {nd }}$ section).

\begin{tabular}{|c|c|c|c|c|c|c|}
\hline Topic & Statements & $\begin{array}{l}\text { Strongly } \\
\text { Disagree }\end{array}$ & Disagree & Agree & $\begin{array}{c}\text { Strongly } \\
\text { Agree }\end{array}$ & $\begin{array}{l}\text { I Don't } \\
\text { Know }\end{array}$ \\
\hline \multirow[t]{3}{*}{ Air Pollution } & S1 & & & & $x$ & \\
\hline & S2 & & $x$ & & & \\
\hline & $\mathrm{S} 3$ & & & $x$ & & \\
\hline \multirow[t]{3}{*}{ Water Pollution } & S4 & & & $x$ & & \\
\hline & S5 & & & $x$ & & \\
\hline & S6 & & & & $x$ & \\
\hline \multirow[t]{3}{*}{ Global Warming } & S7 & & & $x$ & & \\
\hline & S8 & & & & $x$ & \\
\hline & S9 & & & & & $x$ \\
\hline \multirow[t]{3}{*}{ Energy Resources } & $\mathrm{S} 10$ & & & $x$ & & \\
\hline & S11 & & & & & $x$ \\
\hline & $\mathrm{S} 12$ & & $x$ & & & \\
\hline
\end{tabular}

For each topic (i.e., air pollution, water pollution, global warming, and energy resources) the answers were summarized in a unitary area circle. The marks in the axis correspond to each possible answer, i.e., strongly disagree, disagree, agree, strongly agree, and I don't know.

Exemplifying with the statements of the topic air pollution, the answer to S1 was strongly agree and the correspondent area is given by $\frac{1}{3} \times \pi \times\left(\frac{1}{\sqrt{\pi}}\right)^{2}=0.33$, in S2 was chosen the option disagree and the correspondent area is $\frac{1}{3} \times \pi \times\left(\frac{2}{4} \times \frac{1}{\sqrt{\pi}}\right)^{2}=0.08$. Finally, for S3 the answer was agree and the area is $\frac{1}{3} \times \pi \times\left(\frac{3}{4} \times \frac{1}{\sqrt{\pi}}\right)^{2}=0.19$. The total area (i.e., 0.60) is the sum of the partial ones, being the quantitative value regarding the statements of the topic air pollution for participant 1 (Figure 2). The values presented in Table 2 were computed in a similar way, for the remaining topics and for each participant. 

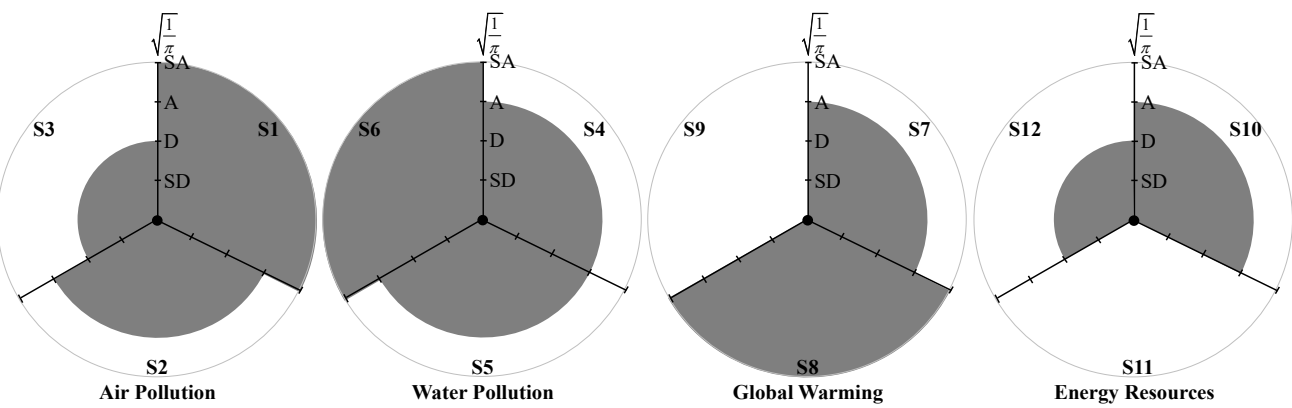

Figure 2. The quantification process of the qualitative information collected in the second section of the questionnaire for respondent 1. SD - Strongly Disagree, D-Disagree, A-Agree, SA-Strongly Agree.

Table 2. A fragment of the knowledge base for the environmental literacy assessment.

\begin{tabular}{ccccc}
\hline Participant & Air Pollution & Water Pollution & Global Warming & Energy Resources \\
\hline 1 & 0.60 & 0.71 & 0.52 & 0.27 \\
$\ldots$ & $\ldots$ & $\ldots$ & $\ldots$ & $\ldots$ \\
147 & 0.56 & 0.85 & 0.56 & 0.08 \\
\hline
\end{tabular}

The values presented in Table 2 were used as inputs of the ANNs models, whereas the output was the assessment of environmental literacy. To achieve the best ANN to assess the environmental literacy several network structures have been elaborated and evaluated (Haykin, 2009).

The performance of ANN models was compared using the confusion matrixes (Fernandes et al., 2020). Among the various topologies tested, the one with the best performance (i.e., higher accuracy) was the 4-3-1 topology (Figure 3).

The respective confusion matrix is displayed in Table 3 (the values displayed correspond to the average of 30 experiments). Based on Table 3, it is possible to compute the model accuracy for training set $(88.1 \%$, i.e., 89 correctly labeled in 101$)$ and for test set $(86.9 \%$, i.e., 40 correctly labeled in 46). Thus, the assessment of environmental literacy through the proposed ANN can be considered satisfactory, exhibiting accuracies higher than $85 \%$. 


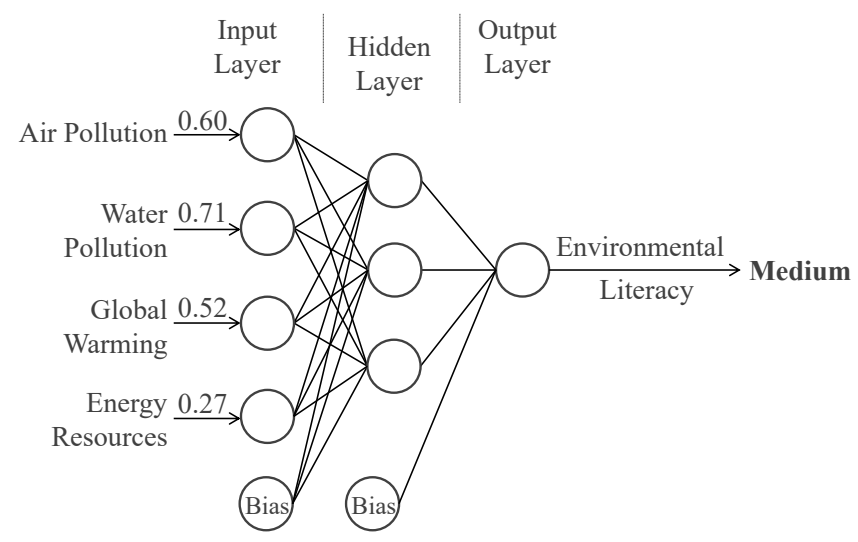

Figure 3. A schematic view of the ANN selected for environmental literacy assessment.

Table 3. Confusion matrix regarding ANN selected for environmental literacy assessment.

\begin{tabular}{lcccccc}
\hline Target & \multicolumn{5}{c}{ Predictive } \\
& Training Set & Test Set & \\
& Reduced & Medium & High & Reduced & Medium & High \\
Reduced & 28 & 4 & 0 & 12 & 2 & 0 \\
Medium & 5 & 50 & 1 & 3 & 22 & \\
High & 0 & 2 & 11 & 0 & 1 & 6 \\
\hline
\end{tabular}

\section{Conclusions and Future Work}

Encouraging environmentally friendly behaviors and developing actions that guarantee the planet's sustainability are mandatory. The population's adhesion can make the actions more effective, and this depends to a great extent on their knowledge on these themes.

This work proposes a methodology to assess the environmental literacy. The results obtained show that the degree of literacy is quite high in relation to the most popular topics in the media, but very low in relation to other topics related to more recent technological solutions, such as the use of biofuels, activated carbon, microorganisms, and biogas. This fact was revealed by the high percentage of answers $I$ don't know obtained in the statements related with those topics. This approach exhibits a satisfactory effectiveness, showing an accuracy higher than $85 \%$.

Furthermore, it allows to recognize the less well-known topics of the population and help the decision makers to promote future dissemination actions as well as their inclusion in the curricula of the various higher education courses. Future work will consider new environmental topics and the use of a cohort of higher education students to study the impact of the scientific area of courses on environmental literacy. 


\section{Acknowledgements}

This work has been supported by FCT - Fundação para a Ciência e Tecnologia within the R\&D Units Project Scope: UIDB/00319/2020.

\section{References}

Disinger, J. F., \& Roth, C. E. (1992). Environmental education research news. The Environmentalist, 12, 165-168.

Fernandes, A., Figueiredo, M., Ribeiro, J., Neves, J., \& Vicente, H. (2020). Psychosocial Risks Assessment in Cryopreservation Laboratories. Safety and Health at Work, 11, 431-442.

Fernandes, A., Vicente, H., Figueiredo, M., Neves, M., \& Neves, J. (2016). An Evaluative Model to assess the Organizational Efficiency in Training Corporations. Lecture Notes on Computer Science, 10018, 415-428. doi: 10.1007/978-3-319-48057-2_29.

Hall, M., Frank, E., Holmes, G., Pfahringer, B., Reutemann, P., \& Witten, I.H. (2009). The WEKA Data Mining Software: An Update. SIGKDD Exploration, 11, 10-18.

Haykin, S. (2009). Neural Networks and Learning Machines. New York: Prentice Hall.

Hollweg, K. S., Taylor, J. R., Bybee, R. W., Marcinkowski, T. J., McBeth, W. C., \& Zoido, P. (2011). Developing a framework for assessing environmental literacy. Washington: North American Association for Environmental Education.

Hungerford, H. R., \& Volk, T. (1990). Changing learner behavior through environmental education. The Journal of Environmental Education, 21 (3), 8-22.

Wackernagel, M., \& Rees, W. (1996). Our ecological footprint: Reducing Human Impact on the Earth. Gabriola Island: New Society Publishers. 